\title{
Thrombocytopenia Absent Radius Syndrome: Rare Cause of Constitutional Thrombocytopenia
}

\author{
Khairoun Chaimae*, Medaghri Alaoui Asmae, KriouilYamna \\ Department of Pediatrics II, Children's Hospital of RABAT, Faculty of Medicine and Pharmacy, Mohammed V \\ University In Rabat, Morocco
}

\begin{abstract}
*Corresponding Author: Khairoun Chaimae, Department of Pediatrics II, Children's Hospital of RABAT, Faculty of Medicine and Pharmacy, Mohammed V University In Rabat, Morocco, Email: chaimaekhairoun1@gmail.com
\end{abstract}

\begin{abstract}
The Thrombocytopenia absent radius syndrome is a very rare congenital malformation of genetic transmission which seems autosomal recessive, characterized by a thrombocytopenia associated with absent rays. The presence of thumb is path gnomonic.

Through our work, we illustrate the case of a 6-month-old infant suffering from syndromic thrombocytopenia. His symptomatology dates back a month before his admission by the appearance of bruises on the face. The clinical examination on admission objectified the presence of conjunctival hemorrhage and petechiae of the forehead, adysmorphia and radial club hands. The malformation assessment objectified minimal hydrocephalus and inter-auricular communication. The blood count showed severe thrombocytopenia responsible for externalized hemorrhage requiring transfusion of the red blood cell and platelet. Genetic confirmation was not done since the mother left against medical advice.
\end{abstract}

\section{INTRODUCTION}

Thrombocytopenia in children is defined by a platelet count below $50 \mathrm{~g} / \mathrm{L}$. Normal values before the age of 15 are, for $95 \%$ of children, between $165 \mathrm{~g} / \mathrm{L}$ and $473 \mathrm{~g} / \mathrm{L}$ with a median value of $299 \mathrm{~g} / \mathrm{L}$ [1]. The discovery of isolated thrombocytopenia most often evokes the diagnosis of immunological thrombocytopenic purpura in children. However, in some cases, other diagnoses such as bone marrow hypoplasia, myelodysplasia, or genetic thrombocytopenia should be considered.

We illustrate through our work, the case of a 6month-old infant suffering from syndromic thrombocytopenia.

\section{CASE REPORT}

This is a 6 month old female infant from nonconsanguineous parents with no specific history. His symptomatology dates back a month before his admission by the installation of bruises on the face. She was referred to our training for suspected leukemia since the presence of a leukocytosis at $73030 \mathrm{E} / \mathrm{mm} 3$ and thrombocytopenia at $14000 \mathrm{E} / \mathrm{mm} 3$.

Clinical examination on admission showed a conscious, non-pyretic infant with conjunctival hemorrhage and petechiae of the forehead. An estimated weight of $5 \mathrm{~kg}(-2 \mathrm{DS})$, a size of $50 \mathrm{~cm}$ (-4DS) and a normal head circumference at $42 \mathrm{~cm}$. we also noticed facial dysmorphia (picture1) with plagiocephaly, hypertelorism, low implanted ears, flattening of the base of the nose, radial club hands without associated anomalies of the fingers (picture 2).

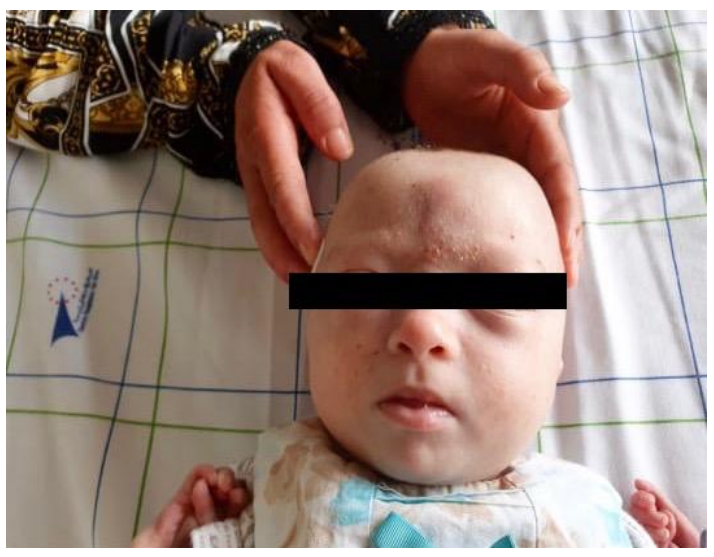

Figure1: Facial dysmorphia

The cardiac auscultation showed the presence of a cardiac murmur in connection with a minimal inter-auricular communication estimated at $6.6 \mathrm{~mm}$. The radiography of the two hands (picture 3) showed bilateral radial agenesis. The rest of the malformation report showed minimal 
hydrocephalus and the absence of an abnormality of the urinary tree and other skeletal anomalies.

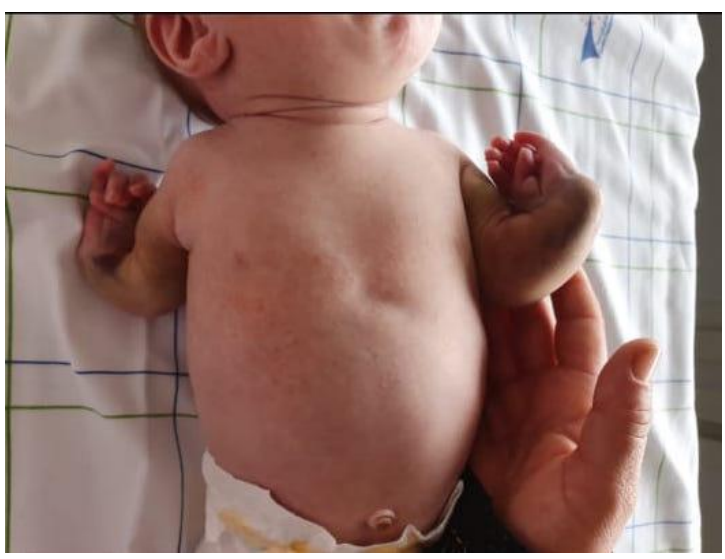

Figure2: Radial club hand

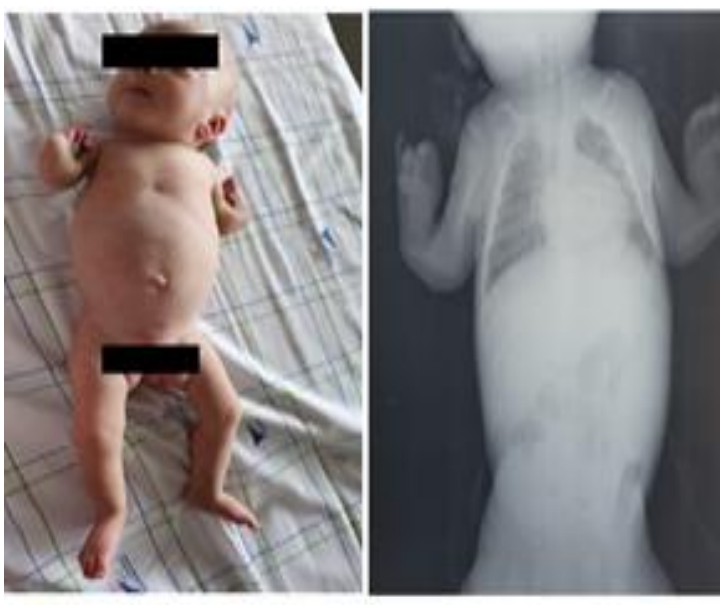

Figure3: bilateral radial agenesis

The biological assessment showed a hemoglobin at $6 \mathrm{~g} / \mathrm{dl}$, VGM at $76 \mathrm{fl}$, TCMH at 22pg, hyperleukocytosis at $73030 \mathrm{E} / \mathrm{mm} 3$ (lymphocytes at $32940 \mathrm{E} / \mathrm{mm} 3$, neutrophlies at $17240 \mathrm{E} / \mathrm{mm} 3$, monocytes at $20010 \mathrm{E} / \mathrm{mm} 3$ ) and a thrombocytopenia at $14000 \mathrm{E} / \mathrm{mm} 3$. The myelogram showed the presence of $3 \%$ of blasts, of a discreet multiline dysmyelopoiesis with monocytosis. The case has been discussed by hemato-oncologists. It is a pre leukemic condition, which required a myelogram check in a month, but the mother left against medical advice.

Therapeutically, the infant was put on a blood and platelet pellet transfusion in the presence of hemorrhagic signs.

Regarding facial dysmorphia, bilateral radial agenesis and thrombocytopenia, we thought of thrombocytopenia of constitutional origin, notably the thrombocytopenia-radial agenesis syndrome. The genetic study was planned but the mother decided to go out against medical advice before genetic confirmation.

\section{DISCUSSION}

Thrombocytopenia absent radius syndrome was first described in 1951[2] and defined as a syndrome in 1969 [3] by Judith Hall. It is a very rare congenital malformation $(<<1 / 100,000$ live births) with a sex ratio of 1 . Its genetic transmission seems autosomal recessive, while an autosomal dominant transmission has also been proposed $[4,5]$.

Clinically, it is characterized by thrombocytopenia associated with absent rays; clinical sign allowing the diagnosis to be made at birth [3]. The presence of the thumb is path gnomonic. Other abnormalities of the skeletal and cardiac systems (23\%) were present, most often a tetralogy of Fallot and septal atrial defects. Genitourinary anomalies including the duplex ureter and horseshoe kidney [3, 6-9] and brain abnormalities "hypoplasia of the cerebella vermis and corpus callosum" have also been reported [10]. In case of our patient, we objectified as anomalies, bilateral radial agenesis, facial dysmorphism, inter-auricular communication and minimal hydrocephalus.

The number of platelets in infants is low, ranging from $10 \times 10^{9} / \mathrm{L}$ to $100 \times 10^{9} / \mathrm{L}$. The bone marrow reveals few megakaryocytes. In the case of our patient, we found severe thrombocytopenia and multiline dysmyelopoiesis with monocytosis, which may be related to a pre leukemic state. In the literature, 4 cases of leukemia have been reported in patients suffering from this syndrome [6-8].

The Thrombocytopenia disappears after childhood, although platelet counts may remain below average until adulthood [11]. The etiology and mode of transmission remain unclear. In all patients, a deletion of chromosome 1q21.1 has been found [12]. However, to this date, the cause and effect link between this deletion and the Thrombocytopenia absent radius syndrome remains unclear. In case of our patient, the karyotype and the genetic study were not done given the discharge of the patient against medical advice.

\section{CONClusion}

The Thrombocytopenia absent radius syndrome is a rare anomaly, which must be evoked in front of any dysmorphia associated with a thrombocytopenia. Mortality during the first year of life is linked to massive haemorrhage, particularly intracranial [5]. Most patients with this syndrome who survived childhood have a normal lifespan $[5,9]$. 


\section{REFERENCES}

[1] G. Biino et al, "Age- and sex-related variations in platelet count in Italy: a proposal of reference ranges based on 40987 subjects' data," (in eng), PLoS One, vol. 8, no. 1, p. e54289, 2013.

[2] W. G. BERNHARD, I. Gore, and R. A. KILBY, "Congenital leukemia," Blood, vol. 6, no. 11, pp. 990-1001, 1951.

[3] J. G. Hall, J. Levin, J. P. Kuhn, E. J. Ottenheimer, and V. McKusick, "Thrombocytopenia with absent radius (TAR)," Medicine, vol. 48, no. 6, pp. 411439, 1969.

[4] S. B. Edelberg, J. Cohn, and N. Brandt, "Congenital Hypomegakaryocytic Thrombocytopenia Associated with Bilateral Absence of the Radius-the TAR Syndrome," Human heredity, vol. 27, no. 2, pp. 147-152, 1977.

[5] V. A. Hedberg and J. M. Lipton, "Thrombocytopenia with absent radii. A review of 100 cases," The American journal of pediatric hematology/oncology, vol. 10, no. 1, p p.51-64, 1988 .

[6] A. Shimamura and B. P. Alter, "Pathophysiology and management of inherited bone marrow failure syndromes," Blood reviews, vol. 24, no. 3, pp. 101-122, 2010.

[7] Z. Fadoo and S. M. Naqvi, "Acute myeloid leukemia in a patient with thrombocytopenia with absent radii syndrome," (in eng), J Pediatr Hematol Oncol, vol. 24, no. 2, pp. 134-5, Feb 2002.

[8] R. S. Go and K. L. Johnston, "Acute myelogenous leukemia in an adult with thrombocytopenia with absent radii syndrome," (in eng), Eur J Haematol, vol. 70, no. 4, pp. 24 6-8, Apr 2003.

[9] B. P. Alter, "Diagnosis, genetics, and management of inherited bone marrow failure syndromes," (in eng), Hematology Am Soc Hematol Educ Program, pp. 29-39, 2007.

[10] A. Skorka, J. Bielicka-Cymermann, D. Gieruszczak-Bialek, and L. Korniszewski, "Thrombocytopenia-absent radius (tar) syndrome: a case with agenesis of corpus callosum, hypoplasia of cerebellar vermis and horseshoe kidney," (in eng), Genet Couns, vol. 16, no. 4, pp. 377-82, 2005.

[11] J. Fiedler et al., "Two patterns of thrombopoietin signaling suggest no coupling between platelet production and thrombopoietin reactivity in thrombocytopenia-absent radii syndrome," (in eng), Haematologica, vol. 97, no. 1, pp. 73-81, 2012.

[12] P. Nurden and A. T. Nurden, "Congenital disorders associated with platelet dysfunctions," (in eng), Thromb Haemost, vol. 99, no. 2, pp. 253-63, Feb 2008.

Citation: Khairoun Chaimae, Medaghri Alaoui Asmae, KriouilYamna. Thrombocytopenia Absent Radius Syndrome: Rare Cause of Constitutional Thrombocytopenia, ARC Journal of Pediatrics. 2020; 6(1):1-3. doi:dx.doi.org/10.20431/2455-5711.0601001.

Copyright: (C) 2020 Authors. This is an open-access article distributed under the terms of the Creative Commons Attribution License, which permits unrestricted use, distribution, and reproduction in any medium, provided the original author and source are credited. 